\title{
Importance of Momentum-Dependent Interactions for the Extraction of the Nuclear Equation of State from High-Energy Heavy-Ion Collisions
}

\author{
J. Aichelin, ${ }^{(1)}$ A. Rosenhauer, ${ }^{(2)}$ G. Peilert, ${ }^{(2)}$ H. Stoecker, ${ }^{(2)}$ and W. Greiner ${ }^{(2)}$ \\ ${ }^{(1)}$ Institut für Theoretische Physik der Universität Heidelberg, D-6900 Heidelberg, Federal Republic of Germany, \\ and Max-Planck Institut für Kernphysik, D-6900 Heidelberg. Federal Republic of Germany \\ ${ }^{(2)}$ Institut für Theoretische Physik, Johann Wolfgang Goethe-Universität, \\ D-600 Frankfurt am Main, Federal Republic of Germany \\ (Received 4 November 1986; revised manuscript received 19 February 1987)
}

\begin{abstract}
We demonstrate that momentum-dependent nuclear interactions (MDI) have a large effect on the dynamics and on the observables of high-energy heavy-ion collisions: A soft potential with MDI suppresses pion and kaon yields much more strongly than a local hard potential and results in transverse momenta intermediate between soft and hard local potentials. The collective-flow angles and the deuteron-toproton ratios are rather insensitive to the MDI. Only simultaneous measurements of these observables can give clues on the nuclear equation of state at densities of interest for supernova collapse and neutron-star stability.
\end{abstract}

PACS numbers: $25.70 . \mathrm{Np}, 21.65 . \mathrm{ff}$

The current studies of high-energy heavy-ion collisions are of broad scientific interest. This is for two reasons: First, they offer a unique testing ground for newly developed methods to study the behavior of strongly interacting quantum systems with finite particle number far from the grounds state. Secondly, one hopes that information on the equation of state (EOS) of dense nuclear matter can be extracted from the experimental data. This knowledge is essential for an understanding of the collapse of supernovae, for neutron-star stability, and for the onset of a possible transition from hadron matter to the quark-gluon plasma.

It has been proposed for many years to use pion production and the collective flow in central high-energy collisions to investigate the nuclear EOS at high density and temperature. ${ }^{1-4}$ The microscopic Vlasov-UehlingUhlenbeck (VUU) theory ${ }^{3-5}$ has exhibited the sensitivity of the pion yields, ${ }^{1,3}$ subthreshold kaon yields, ${ }^{5}$ and the collective flow ${ }^{1,3.4}$ to the nuclear EOS. It has recently been suggested ${ }^{6}$ to test whether this sensitivity to the EOS might be distorted by introduction of momentumdependent interactions (MDI) into the theory. Such interactions had been implemented into the classical "molecular dynamics"7 and the time-dependent mesonfield approach, ${ }^{8}$ which predict ${ }^{7-9}$ a collective sidewards flow in qualitative agreement with the data. ${ }^{10}$

Here we demonstrate the importance of the momentum-dependent interactions using an extension of the VUU approach, dubbed "quantum molecular dynamics" (QMD), ${ }^{11}$ which combines the important quantum features of the VUU theory, namely the Pauli principle, stochastic scattering, and particle production, ${ }^{1,3-5}$ with the long-range $N$-body correlations found in the classical molecular-dynamics method ${ }^{7-9}: N$ Gaussian wave packets with a finite width in configuration and momentum space move on trajectories given by the $\mathrm{N}$-body interac- tion, following Hamilton's equations, $d p / d t=-d H / d q$, $d q / d t=p / m+d U / d p$. Two wave packets can also scatter stochastically at short distances. The width of the wave packets allows for a computation of the phasespace occupancy $(1-f)$ which determines the Pauliblocking probability ${ }^{11}$ of attempted collisions in the same way as in the VUU approach. ${ }^{3-5} \Delta$ 's, pions, and kaons are produced by use of the elementary scattering cross sections in the same way as in the VUU approach. ${ }^{3-5,11}$

The potential $U$ is given by

$$
\begin{aligned}
U=\sum_{i} t_{1} \delta\left(r_{1}-r_{i}^{\prime}\right)+t_{2}\left[\sum_{i} \delta\left(r_{1}-r_{i}\right)\right]^{a} & \\
& +U_{\text {Coul }}+U(\Delta p) \delta\left(r_{1}-r_{i}^{\prime}\right) .
\end{aligned}
$$

The momentum-dependent term $U(\Delta p)$ is taken ${ }^{12}$ from the measured energy dependence of the proton-nucleus optical potential in the range of $10 \mathrm{MeV}<E_{\text {kin }}<1 \mathrm{GeV}$. It can be parametrized as $U(\Delta p)=1.57[\ln (1+5$ $\left.\left.\times 10^{-4} \Delta p^{2}\right)\right]^{2} \rho / \rho_{0}$, where the relative momentum $\Delta p=p_{1}-p_{2}$ is given in units of $\mathrm{MeV} / c$, and $U$ is in $\mathrm{MeV}$. Nuclear-matter calculations ${ }^{12}$ guide the assumed density dependence of $U(\Delta p)$.\} The $d U / d p$ term in the equations of motion yields an effective nucleon mass of $\mathrm{m}^{*} / \mathrm{m}=0.75$ at the Fermi momentum, and $\mathrm{m}^{*} / \mathrm{m}$ $=0.95-0.98$ at relative momenta in the BEVALAC energy region, $E_{\text {lab }}=0.8 \mathrm{GeV} /$ nucleon. The mean potential field can be cast into a form which explicitly shows the density dependence of the interaction in infinite nuclear matter at $T=0$ :

$$
U(\rho)=\alpha \rho / \rho_{0}+\beta\left(\rho / \rho_{0}\right)^{\gamma}+\delta\left\{\ln ^{2}\left[1+\epsilon\left(\rho / \rho_{0}\right)^{2 / 3}\right]\right\} \rho / \rho_{0} .
$$

This can be used to compute the corresponding density dependence of the compressional energy per nucleon (the "cold EOS")..$^{1-3}$ The latter quantity is shown in Fig. 1 for the soft (S) and hard (H) local Skyrme potentials $^{3-5,11}$ and for the interaction with a momentum- 


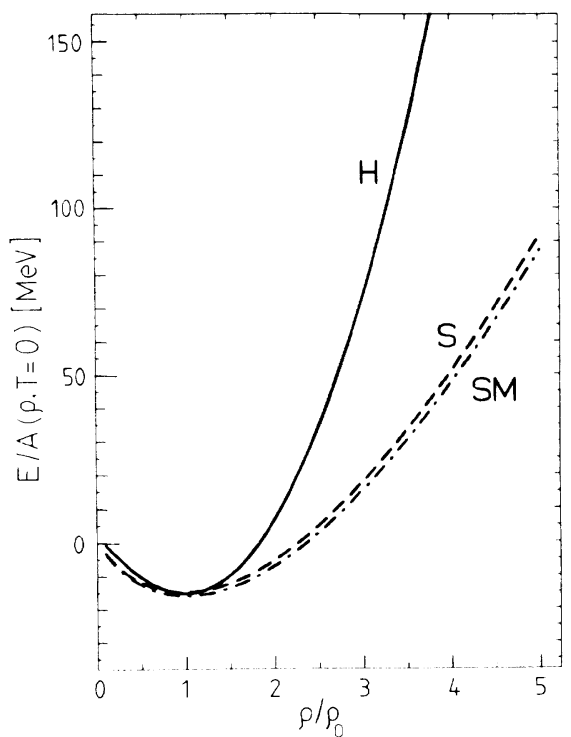

FIG. 1. The density dependence of the compression energy per particle of $T=0$ infinite nuclear matter is shown for the three distinct interactions $\mathrm{S}, \mathrm{SM}$, and $\mathrm{H}$, respectively. Note that the momentum-dependent potential SM exhibits nearly the same energies as the soft local potential $S$. The energy for the hard local potential $\mathrm{H}$ is much higher at the same density.

dependent term, which is hereafter denoted by SM. The parameters $\alpha-\epsilon$ are given by $\alpha=-356,-390,-124$; $\beta=303,320,70.5 ; \gamma=1.166,1.14,2 ;$ and $\delta=1.57$, $\epsilon=21.54$ for the three interactions $\mathrm{S}, \mathrm{SM}$, and $\mathrm{H}$, respectively. Note that $\mathrm{S}$ and $\mathrm{SM}$ lead to nearly the same EOS, which is for infinite matter at $T=0$ much less repulsive than the EOS corresponding to the hard local potential $H$. One would naively expect that the two different interactions $\mathrm{S}$ and $\mathrm{SM}$, which exhibit the same EOS, should yield similar predictions for $4 \pi$ observables in high-energy heavy-ion collisions. However, the main point of this paper is to demonstrate that this is not always the case: Because of the large initial relative momenta of the projectile and target the momentumdependent interaction acts much more repulsively in a collision than in the $T=0$ infinite-nuclear-matter case of Fig. 1 .

Before discussing the influence of the momentumdependent interactions on the $4 \pi$ observables quantitatively, let us first discuss their qualitative effects for the collision dynamics. In the initial penetration phase the effect of the MDI will be most pronounced, since there the separation of the projectile and target in momentum space is the largest. Particles are accelerated by the MDI in the transverse direction early into the reaction. This is different from the sidewards deflection occurring with purely local interactions, ${ }^{1,3}$ which occurs after stopping is achieved and the point of maximum density is reached. Hence the density pileup in the overlap region will be lower when the MDI are present. Consequently, fewer collisions will occur and the observables which are related to this number of collisions, namely particle production rates and longitudinal deceleration, are diminished. The transverse momentum transfer, on the other hand, will be enhanced.

Figure 2 shows the pion, kaon, and deuteron yields for the system La $(0.8 \mathrm{GeV} /$ nucleon $)+\mathrm{La}$ as a function of the impact parameter for the three distinct interactions discussed above. Observe that there is an $\approx 10 \% \mathrm{de}-$ crease of the pion yield as one goes from the soft to the hard local potential, in agreement with earlier findings. ${ }^{3}$ However, the momentum-dependent interactions result in an $\approx 30 \%$ decrease of the pion yield! This strong suppression of the pion yield is due to a $30 \%$ decrease of the number of $N-N$ collisions, which in turn is due to the strong initial repulsion resulting from the large separation of the target and projectile in momentum space. These findings render the proposed determination of the EOS of infinite nuclear matter from the pion yields ${ }^{1-3}$ in heavy-ion collisions alone virtually impossible.

Qualitatively similar, but quantitatively even more dramatic, results are found for subthreshold kaon production, which is of course more sensitive to the details of the reaction dynamics (Fig. 2): The kaon yield drops by a factor of 2 when we go from the soft to the hard local potential, in agreement with the results of Ref. 5, but it drops by a factor of 4 when momentum-dependent terms are introduced, i.e., for the case SM. Again, the lower number of collisions and the deceleration, which is most pronounced for the particles with the highest momenta because of the repulsive MDI, is responsible for this behavior. This subthreshold behavior of the $K^{+}$ production can be useful for an experimental determination of the momentum-dependent part $U(\Delta p)$ of the potential.

Let us now turn to the formation of deuterons, which is calculated in impulse approximation. ${ }^{13}$ The impactparameter dependence of the deuteronlike to protonlike ratio $R$ is shown in Fig. 2. It exhibits nearly the same value $R=0.56$ for the two soft EOS (cases S and SM), while it is about $10 \%$ larger $(R=0.62)$ for the hard EOS, case $H$. Thus the deuteron formation could prove extremely useful to probe the EOS, since it is insensitive to the momentum-dependent interactions for massive systems and directly reflects the EOS of Fig. 1. The entropy per participant nucleon ${ }^{14}$ is found to be similar for the different interactions. Recent high-multiplicityselected experimental data ${ }^{14,15}$ indicate large $R$ values, $(d / p)^{\max } \approx 0.68$ for the system ${ }^{15} \mathrm{La}(0.8 \mathrm{GeV} /$ nucleon $)$ $+\mathrm{La}$. This seems to indicate that the nuclear equation of state is rather hard, in accord with the results on the flow angles discussed below and with the earlier findings, ${ }^{1-3}$ which, however, neglected the momentumdependent terms.

Let us now turn to the collective-flow observables: Figure 3 shows the flow angles and the average trans- 

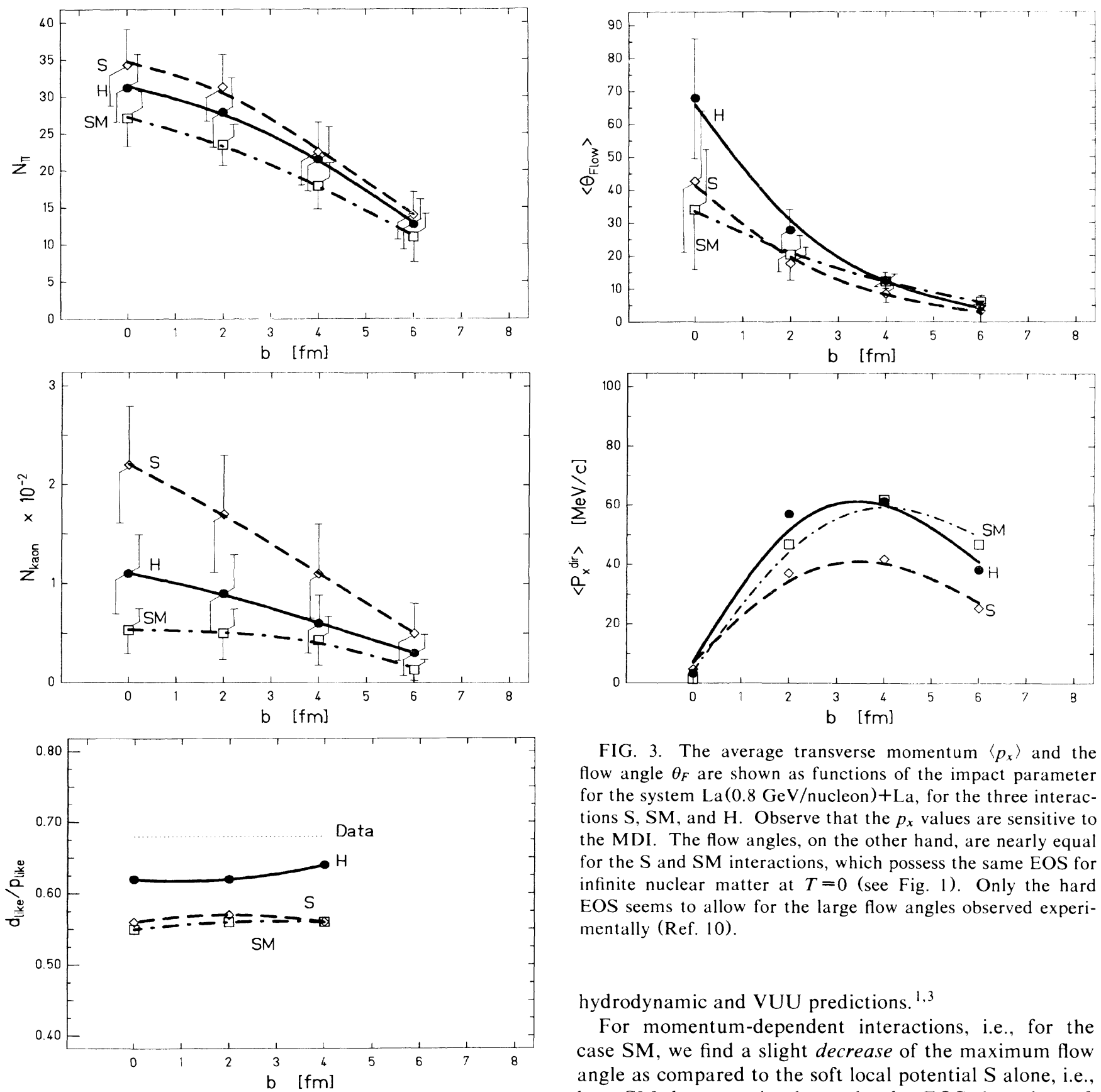

FIG. 3. The average transverse momentum $\left\langle p_{x}\right\rangle$ and the flow angle $\theta_{F}$ are shown as functions of the impact parameter for the system $\mathrm{La}(0.8 \mathrm{GeV} /$ nucleon $)+\mathrm{La}$, for the three interactions $\mathrm{S}, \mathrm{SM}$, and $\mathrm{H}$. Observe that the $p_{x}$ values are sensitive to the MDI. The flow angles, on the other hand, are nearly equal for the S and SM interactions, which possess the same EOS for infinite nuclear matter at $T=0$ (see Fig. 1). Only the hard EOS seems to allow for the large flow angles observed experimentally (Ref. 10).

hydrodynamic and VUU predictions., ${ }^{1,3}$

For momentum-dependent interactions, i.e., for the case SM, we find a slight decrease of the maximum flow angle as compared to the soft local potential $\mathrm{S}$ alone, i.e., here SM does not simulate a harder EOS than the soft local potential! This can be understood as follows: The flow angle is a function of the ratio $\left\langle p_{x}\right\rangle /\left\langle p_{\|}\right\rangle$. The transverse momenta are sensitive to the MDI. The $\left\langle p_{x}\right\rangle$ values for SM and $\mathrm{H}$ are nearly the same and are about double the value obtained with the soft local potential S (Fig. 3). The reduced number of collisions for the case $\mathrm{SM}$, on the other hand, results in a higher longitudinal momentum, which yields nearly the same flow angle for SM as for the soft local potential S. The different contributions of the local potentials and the MDI can be studied by examination of the flow angle and the $p_{x}(y)$

for the system $\mathrm{La}(0.8 \mathrm{GeV} /$ nucleon $)+\mathrm{La}$ : Note that the harder the local potential, the larger is the flow angle. This result of the QMD approach ${ }^{11}$ agrees with previous 
distributions simultaneously. Hence, while no observable is at hand, to date, which can be related directly to the density achieved, the flow-angle distributions seem to be related to the stiffness of the nuclear-matter equation of state. $^{1,3}$ A comparison of the experimental data with the VUU model has yielded evidence for a hard EOS. ${ }^{1,3}$ Experimentally, flow angles by far exceeding the present result using a soft EOS, be it with or without MDI, have been observed. ${ }^{10}$ However, for a quantitative comparison with the experimental data the various fragments ${ }^{11}$ must be subjected to the efficiency cuts of the experimental apparatus, which might have substantial effects on the observables considered.

We have shown that momentum-dependent interactions have a large influence on the $4 \pi$ observables in heavy-ion collisions. Pion and kaon yields seem to be most sensitive to the MDI and could therefore be useful to extract the energy and density dependence of the momentum-dependent part $U(\Delta p)$ (but not the EOS per se) from experiment. From the observed large flow angles and deuteron-to-proton ratios we are tempted to conclude that the EOS is hard. Much work is needed, though, before the nuclear EOS can be pinned down conclusively from experiment.

This work was supported by the Bundesministerium für Forschung und Technologie, Bonn, and the Gesellschaft für Schwerionforschung (GSI), Darmstadt. One of us (J.A.) acknowledges stimulating discussions with Professor J. Huefner.

${ }^{1}$ H. Stöcker, W. Greiner, and W. Scheid, Z. Phys. A 286, 121 (1978); H. Stöcker and W. Greiner, Phys. Rep. 137, 277 (1986).

2J. W. Harris, G. Odyniec, H. Pugh, L. S. Schroeder, M. Tincknell, W. Rauch, R. Stock, R. Bock, R. Brockmann, A. Sandoval, H. Stroebele, R. Renfordt, D. Schall, D. Bangert, J. Sullivan, K. L. Wolf, A. Dacal, C. Guerra, and M. E. Ortiz, Phys. Rev. Lett. 58, 463 (1987); R. Stock, R. Bock, R. Brockmann, J. W. Harris, A. Sandoval, H. Stroebele, K. E. Wolf, H. G. Pugh, L. S. Schroeder, M. Maier, R. Renfordt, A. Dacal, and M. E. Ortiz, Phys. Rev. Lett. 49, 1236 (1982);
J. Harris et al., Phys. Lett. 153B, 377 (1985); R. Stock, Phys. Rep. 135, 259 (1986).

${ }^{3}$ H. Kruse, B. V. Jacak, and H. Stöcker, Phys. Rev. Lett. 54, 289 (1985). Their results disagree with those of G. F. Bertsch, H. Kruse, and S. DasGupta, Phys. Rev. C 29, 673 (1984), who had claimed that there is no sensitivity of the pion yield on the EOS.

${ }^{4}$ J. J. Molitoris and H. Stöcker, Phys. Rev. C 32, 346 (1985), and Phys. Lett. 162B, 47 (1985), and Prog. Part. Nucl. Phys. 15, 239 (1985), and Phys. Rev. C (to be published).

${ }^{5}$ J. Aichelin and C. M. Ko, Phys. Rev. Lett. 55, 2661 (1985). For a comparison between various methods see, e.g., J. Aichelin and H. Stöcker, Phys. Lett. 163B, 59 (1985); J. Aichelin, Phys. Rev. C 33, 537 (1986).

${ }^{6}$ T. L. Ainsworth, E. Baron, G. E. Brown, J. Cooperstein, and M. Prakash, Nucl. Phys. (to be published); C. Gale, G. Bertsch, and S. DasGupta, in Proceedings of the Workshop on the Equation of State, Berkeley, CA, 1986 (to be published), have reported that they find no effect of the MDI on the $p_{x}$ distribution. This is in contrast to the conclusions presented here.

${ }^{7}$ A. R. Bodmer, C. Panos, and A. D. MacKellar, Phys. Rev. C 22, 1025 (1980); L. Wilets, Y. Yariv, and R. Chestnut, Nucl. Phys. A301, 359 (1978).

${ }^{8}$ R. Y. Cusson, P. G. Reinhardt, J. J. Molitoris, H. Stöcker, M. Strayer, and W. Greiner, Phys. Rev. Lett. 55, 2786 (1985).

${ }^{9}$ J. J. Molitoris, J. B. Hoffer, H. Kruse, and H. Stöcker, Phys. Rev. Lett. 53, 899 (1984).

${ }^{10}$ H. G. Ritter et al., Nucl. Phys. C3, A447 (1985); H. A. Gustafsson, H. H. Gutbrod, B. Kolb, H. Loehner, B. Ludewigt, A. M. Poskanzer, T. Renner, H. Riedesel, H. G. Ritter, A. Warwich, and H. Wiemann, Phys. Lett. 142B, 141 (1984); P. Danielewicz and G. Odyniec, Phys. Lett. 157B, 146 (1985).

${ }^{11} \mathrm{~J}$. Aichelin and H. Stöcker, Phys. Lett. 176B, 14 (1986); A. Rosenhauer, J. Aichelin, H. Stöcker, and W. Greiner, J. Phys. (Paris), Colloq. 47, C4-395 (1986), and to be published.

12 J. P. Jeuhenne, A. Lejeune, and C. Mahaux, Phys. Rep. 25 85 (1976)

${ }^{13} \mathrm{~J}$. Aichelin and E. Remler, Phys. Rev. C (to be published), and references therein.

${ }^{14}$ K. G. R. Doss et al., Phys. Rev. C 32, 116 (1985); D. Hahn, and H. Stöcker, Lawrence Berkeley Laboratory Report No. 22378, 1986 (unpublished).

${ }^{15} \mathrm{H}$. Stroebele, Gesellschaft für Schwerionenforschung Report No. 86-10, 1986 (to be published), and to be published; $\mathrm{S}$. Hayashi et al., to be published, and private communication. 